\title{
Long-term analysis of occurrence of malignant lip, oral cavity and pharyngeal cancer in Poland
}

\author{
Wojciech Pinkas' ${ }^{1}$, Melania Brzozowska ${ }^{2}{ }^{3}$, Waldemar Wierzba ${ }^{4}$, Marcin Świerkowski ${ }^{5}$, \\ Adam Kozierkiewicz ${ }^{6}$, Michał Marczak², Ewa Kalinka7 , Mariusz Gujskii , Andrzej Śliwczyński ${ }^{4}, 9$
}

\author{
${ }^{1}$ Department of Pediatric Otolaryngology, Centre of Postgraduate Medical Education, Warsaw, Poland \\ ${ }^{2}$ Department of Management and Logistics in Health Care, Medical University in Lodz, Poland \\ ${ }^{3}$ Department of Drug Management, National Health Fund, Warsaw, Poland \\ ${ }^{4}$ UHE Satellite Campus in Warsaw, University of Humanities and Economics in Lodz, Poland \\ ${ }^{5}$ Military Institute of Medicine, Warsaw, Poland \\ ${ }^{6}$ JASPERS (Joint Assistance to Support Projects in European Regions), Warsaw, Poland
}

${ }^{7}$ Clinical Oncology Unit, Clinic of Oncologic Surgery and Breast Cancer, Polish Mother's Memorial Hospital - Research Institute, Lodz, Poland

${ }^{8}$ Department of Prevention of Environmental Hazards and Allergology, Medical University of Warsaw, Poland

${ }^{9}$ Department of Healthcare Services, National Health Fund, Warsaw, Poland

Introduction. The aim of our study was to analyze the number and structure of patients diagnosed and treated due to cancers of lips, mouth and throat in Poland between 2008 and 2015.

Material and methods. Secondary statistical analysis of data obtained from the Polish National Cancer Registry (KRN) and Polish National Health Fund (NFZ), concerning patients diagnosed for the first time between 2008 and 2015.

Results. Based on the available data, in total there were 30800 new cases of cancers in population of Poland, and 29800 people starting their treatment financed from the National Health Fund.

Discussion. Cancers of the discussed locations, considered individually, are often rare diseases, with the incidence counted in hundreds of people per year in the entire Polish population. Due to the etiology and course, however, they should be analyzed together, which means that they become a significant group.

Conclusions. In recent years, the annual incidence of these tumours was about 4000 cases, $70 \%$ of them were men. There is an approximately $10 \%$ difference in the number of registered cases of these cancers in NFZ databases. The creation of a medical register, including administrative, epidemiological and clinical data, would significantly improve the observation of the population of patients with these tumours.

NOWOTWORY J Oncol 2019; 69, 5-6: 157-162

Key words: lip neoplasms, mouth neoplasms, pharyngeal neoplasms

\section{Introduction}

Neoplasms of the head and neck (H\&N) are classified together, due to their similar aetiology, anatomical pathology and clinical course. It is estimated that they form around $6 \%$ of all cases of cancer and are the reason of $5 \%$ of deaths. Approximately 600000 of new diagnoses of cancer from this group are made worldwide [1-3]. The Cancer Incidence in Five Continents
(CI5) studies demonstrate a worrying increase of incidence in Central Europe [4]. According to the National Cancer Registry (KRN) in Poland, 4389 persons were diagnosed in 2015: 3140 men and 1249 women. The peak incidence is observed at the seventh decade of life, in women it remains at a constant level, starting in the seventh decade of life. According to KRN, the incidence in Poland is higher than average for the other 
European Union countries, approximately 1.4 times higher for men and 1.2 times higher for women. Another source of incidence data for Poland is the National Health Fund (NFZ). This institution is responsible for financing practically all the oncology treatments in Poland. Moreover, it is notified about all the services used by patients both in outpatient and hospital treatment. The goal of the conducted analysis was to compare the incidence and prevalence of head and neck cancers in Poland with health care services utilisation, based on NFZ data from the years 2008-2017.

\section{Materials and methods}

The method used is the secondary statistical analysis of data reported to the national payer - the National Health Fund (NFZ) by medical institutions with an indicated diagnosis of lip, oral cavity and pharyngeal cancer in the years 2008-2017 based on ICD-10 classification of diseases. This method has been already used for similar analyses [5-8]. The analysis included all the reported information on interventions in patients undergoing therapy after a diagnosis of lip, oral cavity and pharyngeal cancer (according to ICD-10 = C00.X - C14.X [9, 10] as the main reason for the intervention; the NFZ databases record all patients whose therapy was financed from the public funds). In order to observe the rules for processing sensitive data, such as personal ID (in Poland PESEL - Universal Electronic System for Registration of the Population), the data were anonymised during the analysis, obtaining the results by processing data sets without sensitive personal data (according to the General Data Protection Regulation) [11]. Indicators of incidence and annual and long-term prevalence were calculated (in the years 2008-2017 - 10-year prevalence and indicator of 10-year prevalence per 100000 inhabitants; in this case the average number of inhabitants of Poland in the years 2008-2017 was used as the denominator). Demographical data (as of 31 December of the given year) used in order to standardise the incidence and prevalence indicators was downloaded from the Central Statistical Office (GUS) website, separately for each year [12].

Additionally, the obtained results were compared to the data established by the National Cancer Registry (KRN - last available KRN report on cancers refers to the year 2015), confirming the treatment of cancer using verification algorithms operating in the NFZ's internal tool - the Disease Treatment Registry (RLC). The principles on which the verification algorithms operate are presented in table I. This has established the level of overdiagnosis of lip, oral cavity and pharyngeal cancer in Poland, this method was already used in analyses [13]. SQL (structured query language) tools were used to extract the data, using a filter in accordance with the ICD-10 diagnoses specified in the assumptions.

\section{Results}

Based on the data for the population of Poland in the years 2008-2017, a total of 120752 diagnoses of lip, oral cavity and pharyngeal cancer were established in men and women. Men constituted a significantly higher percentage of this group 64\%. A total of 46038 patients underwent hospital treatment, of which approx. $72 \%$ were men. The number of patients

Table I. Principles of verification algorithms used in the Disease Treatment Registry (RLC) for oncology

Data on patients diagnosed with a malignant neoplasm by the
RLC system are analysed for:
$\begin{aligned} & \text { 1. Repeatability of the given diagnosis = in order to consider } \\ & \text { a diagnosis established it has to dominate with a given patient and } \\ & \text { be indicated at least } 3 \text { times }\end{aligned}$
2. Indicated diagnostic/therapeutic activities which confirm a cancer
is a situation where a patient with a diagnosed cancer underwent
3. Surgery:
Radiotherapy
Chemotherapy

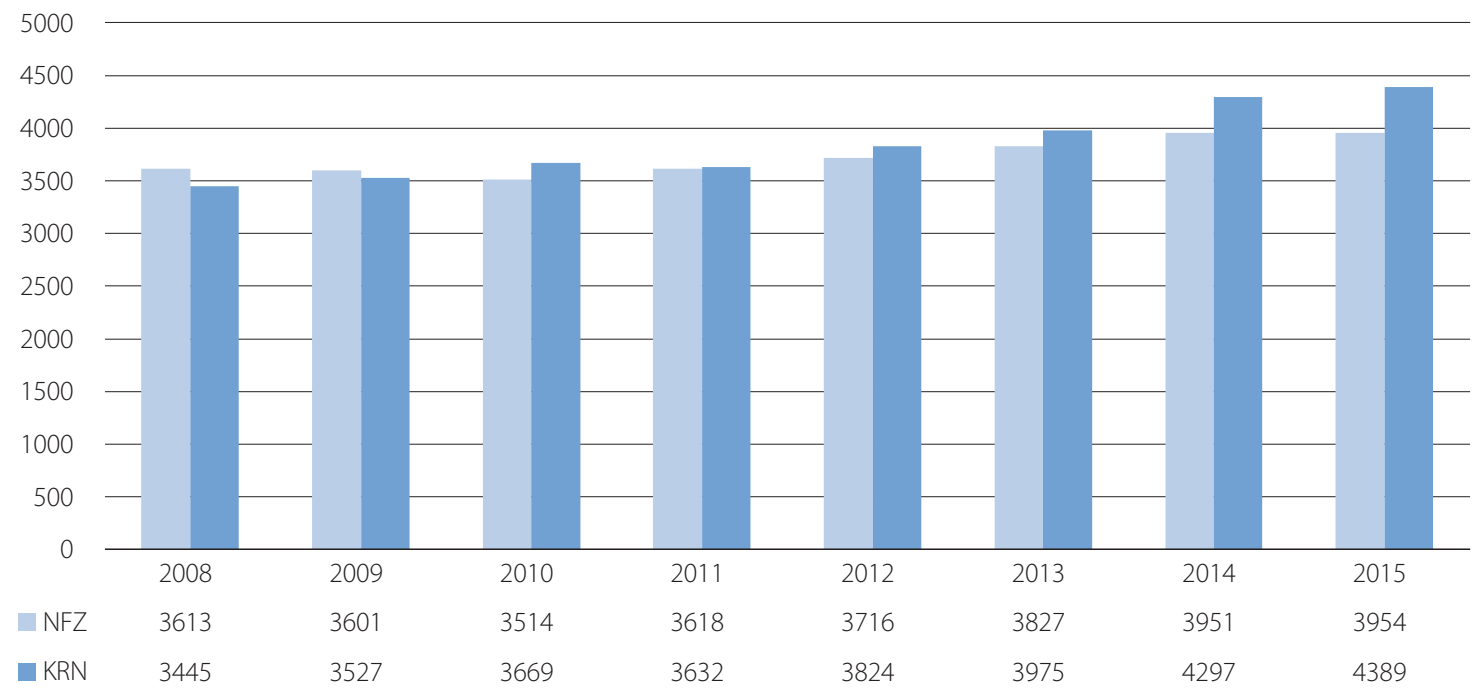

Figure 1. Number of patients registered in KRN and commencing treatment according to RLC 
Table II. Number of patients reported in individual years with a diagnosis of a malignant neoplasm, taking into account their sex and selected type of health care services

\begin{tabular}{|c|c|c|c|c|c|c|c|c|c|c|}
\hline & 2008 & 2009 & 2010 & 2011 & 2012 & 2013 & 2014 & 2015 & 2016 & 2017 \\
\hline \multicolumn{11}{|c|}{ Number of patients in all types of services } \\
\hline 10-year; men & \multicolumn{10}{|c|}{78048} \\
\hline 10-year; women & \multicolumn{10}{|c|}{42704} \\
\hline men & 14413 & 15347 & 15218 & 15147 & 15378 & 15609 & 15942 & 15702 & 15978 & 15928 \\
\hline women & 7519 & 8288 & 7847 & 7910 & 7976 & 8008 & 8279 & 8236 & 8206 & 8412 \\
\hline \multicolumn{11}{|c|}{ Number of patients in hospital treatment } \\
\hline 10-year; men & \multicolumn{10}{|c|}{33094} \\
\hline 10-year; women & \multicolumn{10}{|c|}{12944} \\
\hline men & 4357 & 4141 & 4094 & 3801 & 3706 & 3772 & 3861 & 4627 & 4897 & 4833 \\
\hline women & 1698 & 1655 & 1554 & 1425 & 1478 & 1565 & 1506 & 1774 & 1874 & 2004 \\
\hline \multicolumn{11}{|c|}{ Number of patients in specialist outpatient care } \\
\hline 10-year; men & \multicolumn{10}{|c|}{52546} \\
\hline 10-year; women & \multicolumn{10}{|c|}{25859} \\
\hline men & 10604 & 10706 & 10817 & 11109 & 11440 & 11763 & 12227 & 12107 & 12501 & 12616 \\
\hline women & 5309 & 5358 & 5353 & 5644 & 5738 & 6002 & 6317 & 6182 & 6326 & 6568 \\
\hline
\end{tabular}

Table III. Comparison of KRN and NFZ data for individual types of services

\begin{tabular}{|lccccccccc|}
\hline & 2008 & 2009 & 2010 & 2011 & 2012 & 2013 & 2014 & 2015 \\
\hline KRN & 3445 & 3527 & 3669 & 3632 & 3824 & 3975 & 4297 & 4389 \\
\hline ALL & 21932 & 23635 & 23065 & 23057 & 23354 & 23617 & 24221 & 23938 \\
\hline AOS & 15913 & 16064 & 16170 & 16753 & 17178 & 17765 & 18544 & 18289 \\
\hline SZP & 6055 & 5796 & 5648 & 5226 & 5184 & 5337 & 5367 & 6401 \\
\hline
\end{tabular}

KRN - National Cancer Registry, ALL - the number of patients diagnosed with head and neck cancer (ICD10) in all types of services, AOS - number of patients in specialist outpatient care, SZP - number of patients in hospital treatment

Table IV. Percentage of patients undergoing causal treatment compared to the number of patients reported by medical institutions to the NFZ

\begin{tabular}{|c|c|c|c|c|c|c|c|c|}
\hline & 2008 & 2009 & 2010 & 2011 & 2012 & 2013 & 2014 & 2015 \\
\hline$A L L$ & $15.7 \%$ & $14.9 \%$ & $15.9 \%$ & $15.8 \%$ & $16.4 \%$ & $16.8 \%$ & $17.7 \%$ & $18.3 \%$ \\
\hline AOS & $21.6 \%$ & $22.0 \%$ & $22.7 \%$ & $21.7 \%$ & $22.3 \%$ & $22.4 \%$ & $23.2 \%$ & $24.0 \%$ \\
\hline SZP & $56.9 \%$ & $60.9 \%$ & $65.0 \%$ & $69.5 \%$ & $73.8 \%$ & $74.5 \%$ & $80.1 \%$ & $68.6 \%$ \\
\hline
\end{tabular}

ALL - the number of patients diagnosed with head and neck cancer (ICD10) in all types of services, AOS - number of patients in specialist outpatient care, SZP - number of patients in hospital treatment

indicated in hospital treatment forms $27-28 \%$ of the general number of patients declared by medical institutions, whereas specialist outpatient treatment has registered 78405 patients of patients, including approx. $67 \%$ men. The number of patients indicated in specialist outpatient treatment amounted to approx. $74 \%$ of all indicated patients (Tab. II).

Assuming that the patient's path starts with a suspicion of cancer leading to specialist diagnostics, and then enhanced diagnostics and therapy in hospital treatment, from all patients diagnosed with ICD-10 diagnosis of lip, oral cavity and pharyngeal cancer approx. 74\% end in specialised outpatient care (specialised diagnostics), and approx. 27-28\% end up in hospital treatment (enhanced diagnostics and therapy). Over 10 years the analysed data on the number of patients are highly stable, and the 10-years dynamics is positive and amounts to approx. 1-2\%. A comparison of the number of patients indicated for all types of services, in specialist outpatient care (AOS) and in hospital treatment (SZP) to the number of confirmed cases (KRN) is presented in table III.

The reference source of incidence data in Poland is the KRN, figures of which were compared with the number of new patients treated due to H\&N cancers (Fig. 1). In order to establish the category of "new treated patients" and to enable comparison of the NFZ data with the KRN ones, verification algorithms has been prepared (Tab. I).

Comparison of KRN data and the number of confirmed tumours according to NFZ demonstrates that the data sets are highly conforming. The highest conformity (practically 100\%) occurred in 2011. The data difference between the sets in 2015 amounts to approx. 10\%. Verification algorithms created 
Table V. The annual and periodical (10-year) prevalence indicator for patients reported in individual years with the diagnosis of lip, oral cavity and pharyngeal cancer taking into account sex, undergoing hospital treatment per 100000 province inhabitants

\begin{tabular}{|c|c|c|c|c|c|c|c|c|c|c|}
\hline & 2008 & 2009 & 2010 & 2011 & 2012 & 2013 & 2014 & 2015 & 2016 & 2017 \\
\hline 10-year; men & & & & & & & & & & \\
\hline 10-year; women & & & & & & & & & & \\
\hline men & 24 & 22 & 22 & 20 & 20 & 20 & 21 & 25 & 26 & 26 \\
\hline women & 9 & 8 & 8 & 7 & 7 & 8 & 8 & 9 & 9 & 10 \\
\hline
\end{tabular}

in order to confirm the electronic databases data enable the estimation of the number of patients treated for $\mathrm{H} \& \mathrm{~N}$ cancer with a confirmed diagnosis. By comparing the KRN data and the data reported by medical entities, we can assess the percentage of patients reported to NFZ for whom the occurrence of cancer was confirmed and causal treatment (therapy) was commenced (Tab. IV).

The percentage of patients with a confirmed malignant neoplasm compared to all the patients reported by medical institutions with a H\&N neoplasm demonstrates a growth tendency. This trend is observed both as a total for all types of services, in specialist outpatient care and in hospital treatment. In hospital treatment the highest percentage of patients have a confirmed diagnosis of a malignant neoplasm or commenced therapy (in 2014 this was over 80\% of the patients). Due to the highest compatibility of data between the data reported for hospital treatment and KRN, the standardised indicators and further analyses were calculated based on this type of services (Tab. III). Proportions of 10-year prevalence indicators by gender indicate increased prevalence among 2.73 (men): 1 (women) (Tab. V).

Annual prevalence indicators demonstrate high stability, average per year over the examined period for hospital treat- ment amount to 23/100 $000 \mathrm{M}$ (men); 8/100 $000 \mathrm{~W}$ (women). Prevalence divided into individual groups of diagnoses under hospital treatment is presented in table VI.

Dynamics of the number of reported patients in all types of cancer under analysis since 2012 demonstrates a decreasing tendency. Over the analysed period, the highest number of patients were diagnosed with a malignant neoplasm of other and unspecified parts of the tongue (C02.X $=791.3)$. At the second place in the analysed group there is a malignant neoplasm of the floor of the mouth (CO4.X $=740.8)$, in case of this tumour the dynamics is upwards over the entire analysed period. The third most frequent tumour is the malignant neoplasm of tonsil (C09.X = 724.9). The least frequently diagnosed was the malignant neoplasm of gum (CO3.X = 173.9) and a malignant neoplasm with other and unspecified large glands (C08.X $=175.9)$. Standardisation of data based on the population enables the assessment of the frequency of occurrence of a given neoplasm within the area of Poland and the comparison of the frequency of occurrence of H\&N neoplasms (Tab. VII). Standardised prevalence indicators for the tumours: C00.X from 1.73 (2008) to 1.42 (2017); C11.X from 1.01 (2008) to 0.93 (2017); C14.X from 1.04 (2008) to 0.55 (2017) have a decreasing tendency. Other neoplasms with a high

Table VI. Number of patients in individual years taking into account the ICD-10 group as a part of hospital treatment

\begin{tabular}{|c|c|c|c|c|c|c|c|c|c|c|c|}
\hline & 2008 & 2009 & 2010 & 2011 & 2012 & 2013 & 2014 & 2015 & 2016 & 2017 & Average \\
\hline $\mathrm{COO}$ & 659 & 582 & 564 & 545 & 496 & 481 & 474 & 520 & 544 & 544 & 540.9 \\
\hline C01 & 445 & 419 & 406 & 388 & 325 & 325 & 337 & 394 & 422 & 420 & 388.1 \\
\hline $\mathrm{CO} 2$ & 744 & 771 & 749 & 653 & 675 & 745 & 738 & 884 & 960 & 994 & 791.3 \\
\hline $\mathrm{CO3}$ & 183 & 147 & 134 & 156 & 159 & 168 & 169 & 222 & 195 & 206 & 173.9 \\
\hline CO4 & 687 & 732 & 726 & 679 & 637 & 602 & 683 & 871 & 876 & 915 & 740.8 \\
\hline C05 & 283 & 283 & 285 & 235 & 209 & 234 & 223 & 286 & 291 & 284 & 261.3 \\
\hline C06 & 361 & 354 & 353 & 349 & 389 & 387 & 369 & 429 & 507 & 526 & 402.4 \\
\hline $\mathrm{CO}$ & 418 & 371 & 367 & 317 & 350 & 363 & 342 & 406 & 437 & 417 & 378.8 \\
\hline C08 & 194 & 198 & 188 & 120 & 152 & 158 & 153 & 202 & 180 & 214 & 175.9 \\
\hline CO9 & 755 & 657 & 595 & 563 & 588 & 637 & 645 & 893 & 979 & 937 & 724.9 \\
\hline C10 & 621 & 574 & 586 & 511 & 458 & 476 & 447 & 579 & 589 & 568 & 540.9 \\
\hline C11 & 385 & 449 & 375 & 337 & 319 & 326 & 305 & 301 & 390 & 359 & 354.6 \\
\hline $\mathrm{C} 12$ & 158 & 155 & 189 & 192 & 148 & 163 & 181 & 222 & 249 & 272 & 192.9 \\
\hline $\mathrm{C} 13$ & 741 & 629 & 628 & 609 & 602 & 634 & 614 & 724 & 761 & 743 & 668.5 \\
\hline $\mathrm{C} 14$ & 396 & 316 & 317 & 260 & 244 & 223 & 236 & 241 & 228 & 211 & 267.2 \\
\hline
\end{tabular}


Table VII. Prevalence indicator per 100000 inhabitants taking into account diagnoses in all types of medical services

\begin{tabular}{lllllllllllll} 
& 2008 & 2009 & 2010 & 2011 & 2012 & 2013 & 2014 & 2015 & 2016 & 2017 & Average \\
\hline C00 & 1.73 & 1.52 & 1.48 & 1.41 & 1.29 & 1.25 & 1.23 & 1.35 & 1.42 & 1.42 & 1.41 \\
\hline C01 & 1.17 & 1.10 & 1.06 & 1.02 & 0.85 & 0.85 & 0.88 & 1.03 & 1.11 & 1.10 & 1.02 \\
\hline C02 & 1.95 & 2.02 & 1.96 & 1.69 & 1.75 & 1.94 & 1.92 & 2.30 & 2.50 & 2.59 & 2.06 \\
\hline C03 & 0.48 & 0.39 & 0.35 & 0.40 & 0.41 & 0.44 & 0.44 & 0.58 & 0.51 & 0.54 & 0.45 \\
\hline C04 & 1.80 & 1.92 & 1.90 & 1.76 & 1.65 & 1.56 & 1.78 & 2.27 & 2.28 & 2.38 & 1.93 \\
\hline C05 & 0.74 & 0.74 & 0.75 & 0.61 & 0.54 & 0.61 & 0.58 & 0.74 & 0.76 & 0.74 & 0.68 \\
\hline C06 & 0.95 & 0.93 & 0.92 & 0.91 & 1.01 & 1.01 & 0.96 & 1.12 & 1.32 & 1.37 & 1.05 \\
\hline C07 & 0.51 & 0.52 & 0.49 & 0.31 & 0.39 & 0.41 & 0.40 & 0.53 & 0.47 & 0.56 & 0.46 \\
\hline C08 & 0.51 & 0.52 & 0.49 & 0.31 & 0.39 & 0.41 & 0.40 & 0.53 & 0.47 & 0.56 & 0.46 \\
\hline C09 & 1.98 & 1.72 & 1.56 & 1.46 & 1.53 & 1.65 & 1.68 & 2.32 & 2.55 & 2.44 & 1.89 \\
\hline C10 & 1.63 & 1.50 & 1.53 & 1.33 & 1.19 & 1.24 & 1.16 & 1.51 & 1.53 & 1.48 & 1.41 \\
\hline C11 & 1.01 & 1.18 & 0.98 & 0.87 & 0.83 & 0.85 & 0.79 & 0.78 & 1.01 & 0.93 & 0.92 \\
\hline C12 & 0.41 & 0.41 & 0.49 & 0.50 & 0.38 & 0.42 & 0.47 & 0.58 & 0.65 & 0.71 & 0.50 \\
\hline C13 & 1.94 & 1.65 & 1.64 & 1.58 & 1.56 & 1.65 & 1.60 & 1.88 & 1.98 & 1.93 & 1.74 \\
\hline C14 & 1.04 & 0.83 & 0.83 & 0.67 & 0.63 & 0.58 & 0.61 & 0.63 & 0.59 & 0.55 & 0.70
\end{tabular}

prevalence indicator include: malignant neoplasm of floor of mouth (C.04.X = 1.93) and malignant neoplasm of tonsil (C.09.X = 1.89). Highest value of the indicator amounted to 0.45 patient/100,000 and referred to the malignant neoplasm of gum (CO3.X).

\section{Discussion}

This analysis groups together neoplasms of the structures of the oral cavity, starting from the lip and ending with the laryngeal and nasal parts of the throat. Individual locations are rare sites of neoplasm development: a few dozen to a few hundred cases annually, however as a group these neoplasms form a large category, over 4000 cases annually. Due to their similar aetiology and natural history, it is rational to consider their epidemiology as a whole.

The basic course of information about the incidence of cancer is the KRN, according to which the number of new diagnoses in the last years has exceeded 4300 cases. The NFZ reports record the health services financed from public funds, and based on these reports approximately 4000 of new cases per year were recorded. The difference, sometimes up to $10 \%$, may be caused by various methods used to create these sets. The differences may also result from the fact that the clinical differentiation of the initial point of the neoplasm may be problematic, in particular when the neoplasm is clinically advanced in a given location. Also blurred categories, like C14 malignant neoplasm of other and ill-defined sites, C06 malignant neoplasm of other and unspecified parts of mouth may be used interchangeably with the codes of more precise categories. Cancers of H\&N are much more frequently present in men; approximately over $70 \%$ of patients are male. A similar relation of men to women was demonstrated by studies based on the database of the International Agency for Research on Cancer [14]. Cancer in men more frequently occurs in the structures in the rear of the nose and throat cavity, like laryngeal or mouth part of the throat, the same incidence in men and women occurs only for the neoplasms of large salivary glands. The percentage of patients with whom the therapy is not continued, present on all the levels of care, should be assessed in a positive manner, as a manifestation of the care exhibited by medical professionals related to the suspicion of cancer, however, since this percentage is stable over the entire period under analysis, it seems justified to suggest to medical personnel, in particular to laryngologists, a continuous and ongoing education in the field of cancer diagnostics and therapy.

\section{Conclusions}

Despite different methods of data collections and their purpose, both the NFZ and the KRN data demonstrate similar incidence of lip, oral cavity and pharyngeal cancers. In the years 2008-2017, a total of 46000 cases of lip, oral cavity and pharyngeal cancer were reported in hospital treatment, including approx. 33000 in men and approx. 13000 in women; annual average of 4200 men and 1600 women. Men constituted a higher percentage of patients; approx. $64 \%$ in all categories in total, in hospital treatment - $72 \%$, in specialist outpatient care $-67 \%$. Of all patients reported by medical institutions with ICD-10 diagnoses of lip, oral cavity and pharyngeal cancer approx. 74\% end up in specialist outpatient care (specialised diagnostics) and approx. 27-28\% end up in hospital treatment (enhanced diagnostics and therapy).

Conflict of interest: none declared 


\section{Wojciech Pinkas}

Centre of Postgraduate Medical Education

Department of Pediatric Otolaryngology

ul. Cegłowska 80

01-809 Warszawa, Poland

e-mail:wj.pinkas@gmail.com

Received: 12 Jun 2019

Accepted: 11 Sep 2019

\section{References}

1. Marur S, Forastiere AA. Head and neck squamous cell carcinoma: update on epidemiology, diagnosis and treatment in Mayo Clinic Proceedings. Elsevier. 2016; 91 (3): 38-396.

2. Rettig EM, D'Souza G. Epidemiology of head and neck cancer. Surgical Oncology Clinics. 2015; 24 (3): 379-396.

3. Gupta B, Johnson N, Kumar N. Global epidemiology of head and neck cancers: a continuing challenge. Oncology. 2016; 91 (1): 13-23.

4. Simard EP, Torre LA, Jemal A. International trends in head and neck cancer incidence rates: differences by country, sex and anatomic site. Oral Oncology. 2014; 50 (5): 387-403.

5. Didkowska JA, Wojciechowska U, Kozierkiewicz A et al. Comparison of data sets from National Cancer Register and National Health Fund based on the example of breast and colorectal cancers. Oncology in Clinical Practice. 2012; 8 (4): 129-142.
6. Śliwczyński A, Tkacz A, Kowalski A et al. Nowotwory złośliwe jelita grubego w świetle danych Narodowego Funduszu Zdrowia w latach 2006-2009. Nowotwory. Journal of Oncology. 2011; 61 (3): 252-252.

7. Wierzba W, Śliwczynski A, Karnafel W et al. Comparison of the incidence of new malignancies in diabetic patients in urban and rural populations in Poland in the years 2008-2014 based on the database of the National Health Fund. Archives of Medical Science. 2017; 15 (2): 330-336.

8. Śliwczyński AM, Brzozowska M, Teter Z et al. Regional differences in the frequency of diabetes occurrence and its treatment costs during the years 2008-2013, based on the NFZ (National Health Fund) database. Archives of Medical Science. 2017; 13 (1): 256.

9. 26 June 2018 Regulation of the Minister of Health changing the regulation on the scope of necessary information collected by service providers, detailed method of recording such information and its provision to institutions required to finance the services from public funds (Dz. U. of 2018 item 1257)

10. Regulation 63/2018/DI on establishing a detailed XML reporting message for outpatient and hospital services.

11. Personal Data Protection Act of 10 May 2018 (Dz. U. of 2018 item 1000).

12. SWAID. http://swaid.stat.gov.pl/SitePagesDBW/Demografia.aspx

13. Kozierkiewicz A, Jach R, Basta T et al. Efficacy and costs of ovarian cancer therapy in Poland - regional approach. Ginekologia Polska. 2015; 86 (8).

14. Shield KD, Ferlay J, Jemal A et al. The global incidence of lip, oral cavity, and pharyngeal cancers by subsite in 2012. A Cancer Journal for Clinicians. 2017; 67 (1), 51-64. 